\title{
An Efficient Registration Algorithm for Advanced Fusion of 2D/3D Angiographic Data
}

\author{
Martin Groher ${ }^{1}$, Ralf-Thorsten Hoffmann ${ }^{2}$, Christoph Zech ${ }^{2}$, \\ Maximilian Reiser ${ }^{2}$, Nassir $\mathrm{Navab}^{1}$ \\ ${ }^{1}$ Lehrstuhl für Informatikanwendungen in der Medizin, TU München \\ ${ }^{2}$ Klinische Radiologie des Klinikums Grosshadern, LMU München \\ Email: groher@cs.tum.edu
}

\begin{abstract}
Computed tomography angiography (CTA) is often used for pre-interventional diagnosis and planning, whereas nowadays, mostly 2D angiograms are acquired for intra-interventional catheter guidance. Spatial information from pre-interventional scans is not transferred to the intervention yet since existing 2D-3D registration methods either require a good initial manual alignment or have rather long runtime and thus lack clinical usefulness. We propose a fast and automatic method for 2D-3D registration and evaluate methods for intra-interventional visualization and navigation. Moreover, we introduce an easy clinical workflow for transferring a planned roadmap from pre-interventional 3D to intra-interventional 2D using the registration. We demonstrate the good quality of fit and the fast runtime of the algorithm on one phantom and three patient data sets.
\end{abstract}

\section{Introduction}

Catheterizations are carried out on a daily basis in many hospitals. Very often, a 3D pre-interventional computed tomography angiography (CTA) is acquired for pathology detection and procedure planning. During the treatment, only 2D projections (e.g. digitally subtracted angiography, DSA) are acquired for navigating the catheter to the region of interest. A registration and proper visualization of pre- and intra-interventional data during the procedure would help the physician to navigate the catheter through the vessel system. We propose a registration algorithm whose runtime is only depended on the number of junctions in the $3 \mathrm{D}$ vasculature (mostly 20-30) and thus outperforms most other 2D-3D registration algorithms based on non-linear optimization (see $[1,2,3,4]$ for the most recent methods).

\section{Method}

Rigid 2D-3D registration aims at recovering the 6 degrees of freedom (DOF) providing the viewing parameters of $2 \mathrm{D}$ image capture. The 6 DOFs are also referred to as the extrinsic parameters $[R \mid t]$ of a perspective projection,

$$
x=P X=K[R \mid t] X
$$


$P \in \mathbb{R}^{3 \times 4}$ projects a homogeneous $3 \mathrm{D}$ point $X$ onto a homogeneous $2 \mathrm{D}$ point $x$ and can be decomposed into $K$ (intrinsic parameters) and rotation $R$ and translation $t=\left(t_{x}, t_{y}, t_{z}\right)^{T}$ (extrinsic parameters).

In order to produce feature coordinates to register, we segment the arterial vasculature in $3 \mathrm{D}$ from an angiographic phase of a CTA and in $2 \mathrm{D}$ from a DSA using region growing. We then extract centerline graphs with a thinning and wave propagation algorithm [5]. Only the bifurcations in the 3D and 2D data sets are used as features for registration. State-of-the-art angiographic C-arm devices store lots of information concerning imaging geometry. Each 2D angiogram is provided with the calibration matrix $K$, a source-to-object distance $\left(\mathrm{STO}^{1}\right)$, and a source-to-detector distance (STD). Moreover, a primary and secondary rotation angle provide a rather decent estimate of the rotation matrix $R$.

We use all this information to produce initial image as well as object coordinates of the extracted vessel trees. For all node coordinates of the $2 \mathrm{D}$ centerline graph $\tilde{x}_{i}$ we undo the transformation specific to the imaging device

$$
x_{i}=K^{-1} \tilde{x}_{i}
$$

For all node coordinates of the $3 \mathrm{D}$ centerline graph $\tilde{X}_{i}$, we apply an initial transformation including primary/secondary angle in $\tilde{R}$ and an approximate ztranslation $\tilde{t}=(0,0, \mathrm{STD}-\mathrm{STO})^{T}$

$$
X_{i}=\tilde{X}_{i} \tilde{R}+\tilde{t}
$$

\subsection{Registration of $t_{x}$ and $t_{y}$}

In order to find a value for $\mathrm{x}$ - and $\mathrm{y}$-translation we assume rotation and $\mathrm{z}$ translation given and try to establish correspondences between bifurcations using translation-invariant feature descriptors. From the different descriptors proposed in the literature, we have chosen the shape context descriptor of Belongie et al [6] since it describes features by their relative translation to all other features in the same shape and is independent of intensity neighborhoods. This inherent translational invariance is followed by a rotational invariance for small rotations which holds for our scenario. For each bifurcation of the 2D and projected 3D graph we create a histogram including all translation vectors to all other nodes of the graph (bifurcations and sampling nodes of vessel segments) binned over log-polar coordinates. To find a corresponding bifurcation to $v_{i}$ in one graph, we determine the minimal "distance" $d_{\min }$ between histograms $h_{i}, h_{j}{ }^{2}$ of all bifurcations $v_{j}$ in the other graph

$$
d_{\min }=\min _{j} \sum_{k}\left(h_{i}(k)-h_{j}(k)\right)^{2}
$$

\footnotetext{
${ }^{1}$ The STO is actually a source-to-table distance, but is called source-to-object distance in the DICOM header

${ }^{2}$ the histograms contain normalized polar coordinates
} 
where $k$ is the number of bins in each histogram. Here, we do not require a oneto-one correspondence solution (i.e. one feature might correspond to more than one feature in the other graph). We allow this inexact modelling of the correspondence problem because we just try to find one best correspondence and discard the others. This implementation speeds up the matching process compared to the original implementation using a bipartite graph matching algorithm.

Since there are many false matches due to segmentation errors, overlay, and deformation changes, we try to iteratively find one "best" correspondence between two bifurcations to receive $\mathrm{x}$ - and $\mathrm{y}$-translations. For that, we iteratively translate the $3 \mathrm{D}$ graph in $x$ - and $y$-direction parallel to the image plane such that a projected $3 \mathrm{D}$ bifurcation is laid over its corresponding $2 \mathrm{D}$ bifurcation. A cost function $f_{\text {topo }}$, described in [1], is evaluated and the $(x, y)$-translation yielding the lowest value is chosen. ${ }^{3}$ This search algorithm for the best $(x, y)$-translation is linear in the number of bifurcations in the 3D graph (mostly between 20 and 50 ) and thus requires only a small and fixed number of cost function evaluations, which results in a speed-up compared to other registration algorithms based on non-linear optimization.

\footnotetext{
${ }^{3} f_{\text {topo }}$ uses geometric distances between bifurcations as well as topological information
} to rate the similarity between $3 \mathrm{D}$ and $2 \mathrm{D}$ graph

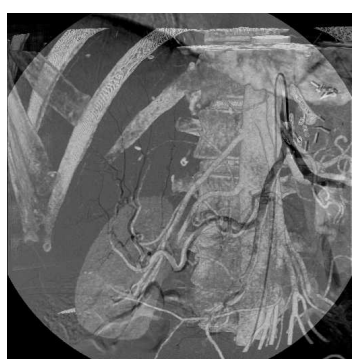

(a) 2D-3D overlay

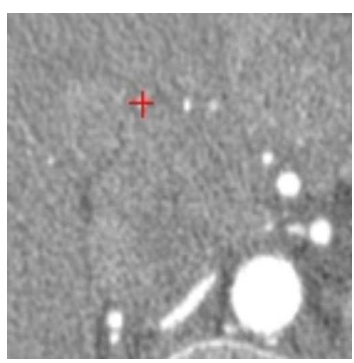

(d) Planned destination

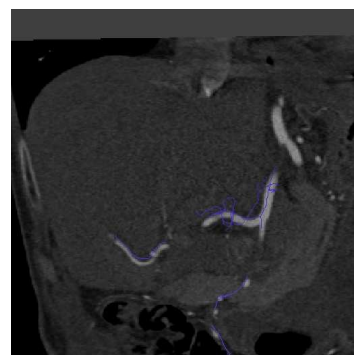

(b) MPR

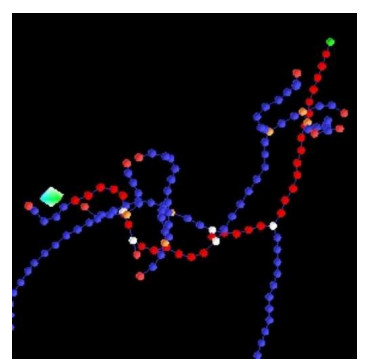

(e) 3D roadmap

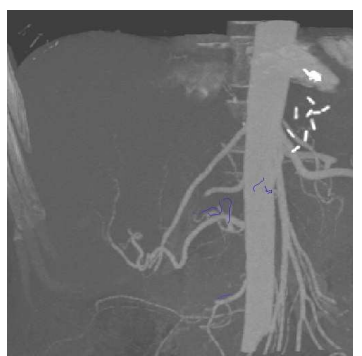

(c) MIP

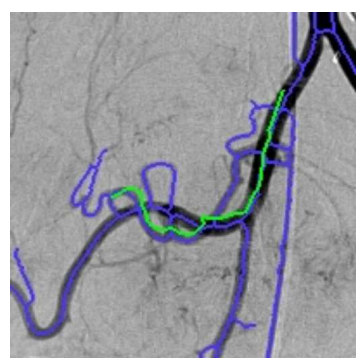

(f) Projected roadmap

Fig. 1. Intra-interventional visualization and navigation 


\section{Experiments and results}

We have evaluated our approach on a rigid phantom for which a ground truth pose was created through intensity-based registration. Moreover, we asked physicians to register the patient data sets manually. In all cases, the pose could be recovered within 2-6 seconds. Deviations from the ground truth registration were in the range of $0.3 \mathrm{~mm}$ for $\mathrm{x}$ - and $\mathrm{y}$-translations, $10 \mathrm{~mm}$ for $\mathrm{z}$-translation, and $<5^{\circ}$ for rotations. We want to emphasize that a better accuracy could be reached (e.g. with a non-linear optimization taking this method as initialization step), but the goal of intra-interventional visualization and road- mapping only requires a rough alignment according to physicians. We therefore discarded the optimization for the sake of speed and thus intra-interventional usability.

\subsection{Intra-interventional visualization and navigation}

Once registered, the pre-interventionally acquired 3D CTA data can be transferred to the interventional room for improved orientation, navigation, and roadmapping. We have shown two different visualization techniques to physicians asking for their feedback. Moreover, a simple planning tool for the pre-interventional data was implemented that allowed physicians to create a $3 \mathrm{D}$ roadmap before the intervention. After the registration process this roadmap can be projected onto the current DSA highlighting the vessel path the catheter should take to reach the region of interest.

Direct volume rendering can be used to project the $3 \mathrm{D}$ data from the same viewpoint as the current DSA image. The resulting $2 \mathrm{D}$ image can be blended over the actual 2D DSA for 2D-3D overlay (fig. 1(a)). The fused images are shown on one monitor as an "In-place visualization" [7].

Multi-planar reconstruction (MPR) is a rendering technique to display a $2 \mathrm{D}$ slice of the volume with an arbitrary cutting plane. On a second monitor, we offer a cutting plane visualizing a slice perpendicular to the viewing direction in the middle of the volume. A slider translates the cutting plane along the viewing direction (fig. 1(b)). Since vessels have a much brighter intensity than surrounding tissue we allow maximum intensity projections (MIP) from partial volumes, too (fig. 1(c)). If the partial volume is properly defined, a manual browsing through slices can be avoided.

According to physicians the 2D-3D overlay on one view does not improve $3 \mathrm{D}$ perception in the interventional room. The $3 \mathrm{D}$ volume is projected and, since the registration information is to be kept, should not be transformed and seen from any other viewpoint. Thus, the overlay is only giving additional $2 \mathrm{D}$, but not 3D perception. The MPR visualization was favored because it resembles the rendering of orthogonal slices radiologists are used to from diagnostic procedures. Moreover, if shown on a second monitor, it does not influence the 2D DSA and can be used as general orientation whereas fine-grained navigation is done on fluoroscopic/DSA images. By showing different slices and/or MIPs a 3D perception can be created. Although In-place visualization of registered data is 
preferred when fusing different 3D data sets [7], in the case of 2D-3D data fusion out-of-place visualization proves to be the better choice.

\section{$3.2 \quad$ Roadmapping tool}

With the roadmapping tool, an interventionalist can plan a procedure preinterventionally on the CTA data by simply clicking on the vessel branch to be embolized (fig. 1(d)). A shortest path is automatically calculated using the segmented vasculature's centerline. The path is followed to the main vessel by increasing vessel diameter. This roadmap can be visualized on the extracted 3D vasculature for orientation (fig. 1(e)) and projected with the registration parameters onto the current 2D image for improved catheter navigation (fig. $1(\mathrm{f})$ ). This planning feature received very positive feedback from physicians since navigation through the vessel system can be significantly improved by roadmap projection.

\section{Discussion}

We introduced a fast 2D-3D registration algorithm working on junctions of vessel systems which is the only visible structure in catheter interventions. We are well aware that the resulting registration parameters are only near to an optimal solution. However, they are sufficient for the physician to be able to discern vessels and for the system to project a planned roadmap. Moreover, we evaluated different visualization techniques and proposed a clinical workflow for interventional planning in $3 \mathrm{D}$ and $2 \mathrm{D}$ roadmapping. We received a positive feedback from physicians and will now focus on a more extensive clinical study to further evaluate the method.

\section{References}

1. Groher M, Padoy N, Jakobs TF, Navab N. New CTA protocol and 2D-3D registration method for liver catheterization. LNCS 2006;4190:873-881.

2. Jomier J, Bullitt E, v Horn M, Pathak C, Aylward SR. 3D/2D model-to-image registration applied to TIPS surgery. LNCS 2006;4191:662-669.

3. Florin C, Williams J, Khamene A, Paragios N. Registration of 3D angiographic and x-ray images using sequential monte Carlo Sampling. LNCS 2005;3765:427-436.

4. Turgeon GA, Lehmann G, Guiraudon G, et al. 2D-3D registration of coronary angiograms for cardiac procedure planning and guidance. Med Phys 2005;32:37373749 .

5. Zahlten C, Jürgens H, Peitgen HO. Reconstruction of branching blood vessels from CT-data. In: Eurographics Workshop of Visualization in Scientific Computing. Springer; 1994. 161-168.

6. Belongie S, Malik J, Puzicha J. Shape matching and object recognition using shape contexts. IEEE Trans PAMI 2002;24:509-522.

7. Tory M. Mental registration of $2 \mathrm{D}$ and $3 \mathrm{D}$ visualizations: An empirical study. In: Procs IEEE Visualization Conference. Seattle, Washington, USA; 2003. 49. 